\title{
Requirements for Fast Particle Measurements on ITER and Candidate Measurement Techniques
}

\author{
F P Orsitto 1), J-M Noterdaeme 2), A E Costley 3), A J H Donné 4), and the ITPA TG on \\ Diagnostics*
}

1) ENEA-EURATOM Association C R Frascati 00044 Frascati(Italy);

2) Max Planck IPP Garching $(\mathrm{Ge})$;

3)ITER IT, Naka Joint Work Site (JA);

4) FOM-Rijnhuizen TEC,Nieuwegein(NL)

(*) Membership listed in ref 33 .

e-mail contact of main author: orsitto@frascati.enea.it

\begin{abstract}
Recent JET and JT-60U results, have underlined the need to look into the measurement requirements of fast particles diagnostics in ITER. Not only alpha particles, but all fast ions $\left(\mathrm{He}^{4}, \mathrm{p}, \mathrm{D}, \mathrm{T}, \mathrm{He}^{3}\right)$ present in the plasma should be diagnosed. Furthermore, the electron diagnostics, monitoring the deviations from a Maxwellian distribution function, play a central role in the Electron Cyclotron Heating and Current Drive.In the present ITER measurement requirements only alpha particles and $\mathrm{He}$ ions are mentioned: space resolution is $\delta \mathrm{r}=\mathrm{a} / 10$ ( $\mathrm{a}$ is the minor radius), time resolution is $\delta \tau=100 \mathrm{~ms}$, accuracy $10-30 \%$. The new proposed measurement requirements for fast particles are: spatial resolution $\sim \mathrm{a} / 20$ (10 cm on ITER); time resolution (minimum) $\sim 100 \tau_{\mathrm{A}}(\sim 100 \mu$ s for ITER); density of fast ions: the minority ions could be $4-10 \%$ of the plasma density. The candidate diagnostic techniques considered for ITER are amongst others : $\gamma$-ray spectroscopy; fast ion Collective Thomson Scattering (CTS); Charge Exchange Recombination Spectroscopy (CXRS). $\gamma$-ray spectroscopy detects ions in the energy range $1<\mathrm{E}<5 \mathrm{MeV}$, and $\mathrm{R} \& \mathrm{D}$ is needed to demonstrate the feasibility of these measurements in the presence of a neutron background. Techniques using ultraviolet spectroscopy of ions like Krypton are being tested, for the low energy part of spectrum of fast particles. A design study of CTS shows that the requirements are close to be met, the system includes a high power gyrotron operating in a region not yet tested in current applications (i.e. using a gyrotron at a frequency below the first ECE harmonic).For CXRS the spatial resolution and accuracy are not achievable for $\mathrm{r} / \mathrm{a}<0.3$, and $\mathrm{R} \& \mathrm{D}$ is needed to assess the minimum figures in terms of accuracy possible for measurements close to the centre. The measurement requirements deduced from the need of detecting the effects of interaction of fast particles with MHD instabilities put important new objectives to the candidate diagnostic systems. In particular diagnostics for fast particles in the energy region $200<\mathrm{E}<1300 \mathrm{keV}$ and diagnostics for escaping fast particles need a urgent development.
\end{abstract}

\section{Introduction}

Recent developments in physics results of interactions of MHD instabilities and fast ions on JET and JT-60U[1-5], have underlined the importance of looking into the parameter measurement requirements of fast particles diagnostics for ITER because of the strong impact of fast particle behaviour on the various ITER scenarios.

In general fast ions ( alpha particles ) generated by D-T reactions, or driven by neutral beam ( $\mathrm{D}$ or $\mathrm{H}$ ions) and minority fast wave current drive ( minority $\mathrm{He}^{3}$ ) can generate instabilities, resonating with fast ions. These instabilities can degrade the confinement of those fast ions, leading ultimately to their loss and a damage of plasma facing components. Monitoring the dynamics of all fast ions can be important for the understanding of energy transfer between fast ions and bulk plasma. Ultimately the aim is to prevent the damage of the device.

A comparison of the measurement requirements with the envisaged capability of the planned fast particle diagnostic systems on ITER is required to guide the developments of the diagnostic systems to get closer to the realization of the accuracy and resolutions( time and space). The aim is 
to produce data useful for an optimized evaluation of ITER scenarios. In practice only an integrated data analysis could give a complete information on the behaviour of fast particles: one of the aim of the present paper is to develop a complete overview of the capabilities of various diagnostic systems available, defining clearly their complemetarity.

The present paper contains the following proposals and related justifications, to upgrade the measurement capability of ITER:

i) all fast ions $\left(\mathrm{He}^{4}, \mathrm{p}, \mathrm{D}, \mathrm{T}, \mathrm{He}^{3}\right)$ present in the plasma, (not only alpha particles), including ions used for minority heating, should be diagnosed, because all of them can interact with plasma instabilities.

The physics of the interaction between plasma instabilities and fast ions leads to a revision of requirements on measurements in terms of space and time resolution.

ii) Ideally the wave propagation inside the plasma could be diagnosed for a better understanding of the energy transfer process to minority ions. The diagnostics of waves propagating inside plasma and interacting with fast ions, like interferometry and reflectometry, have given a real step foreward to the clarification of important details of the (Alfvén ) wave dynamics.

iii) The diagnostics for measuring the parameters of electron distribution function must be included as well, to monitor using ECE ( or a combination of ECE and Thomson Scattering ) the deviations from a Maxwellian distribution function, which play a central role in the dynamics of Electron Cyclotron Heating and Current Drive.

In Trace Tritium Experiments on JET [1] evidence of fast ion transport due to Toroidal Alfvén Eigenmodes(TAE) [2] and fishbone [3] modes were clearly detected. In advanced regimes on JT 60U [4,5], Abrupt Large-Amplitude Events (ALE) were observed where, as a consequence of interaction with Energetic Particle modes, a spatial redistribution of fast ions was demonstrated. Energetic Particle modes(EPM)[6] were related with ALE in recent theoretical papers [7]. Interactions of TAEs with alpha particles were already documented on TFTR [8] and JET first campaign in Deuterium-Tritium DT1 [9]. Fast particle driven TAEs were studied on Alcator CMod and DIIID [10,15], where the effect of TAE on the confinement of fast ions was clearly documented.

Fast particle losses can be due also to a magnetic field ripple and experiments were done recently on JFT-2M [4] demonstrating an increase of heat flux on the first wall due to energetic ion losses when the ripple was increased. Earlier experiments on JET [9,17] found that particles of intermediate energy $10-50 \mathrm{keV}$ were mainly lost, instead of energetic ions. A study made for ITER [18] concluded that an alpha particle loss higher than 5\% could be damaging for ITER first wall.

The instruments used at present to diagnose the fast ion confinement in a number of present experiments are : i) $\gamma$-ray spectroscopy [13] ; ii) neutron cameras with fast electronics for discrimination between neutrons and gammas [5] ; iii) infrared O-mode interferometry [3,14] ; iv) fast magnetics ( Mirnov coils ); v) neutral particle analizers [5] ; vi) $\mathrm{D}_{\alpha}$ emission [15] ; vii) Natural diamond spectrometers [16] ; and vii) fast ion Collective Thomson Scattering, which was carried out on TEXTOR device [19] .

Diagnosing fast particles is not just the use of a single diagnostic, but the integration of a number of diagnostics to obtain insight in the dynamics of fast particles in ITER. The integration of a system of diagnostics was experienced at JET during the first DT experiment and the more recent Trace Tritium Experiment. Furthermore a redundancy in the measurements is needed, to reduce uncertainties.

In the present measurement requirements [12], only alpha particles and $\mathrm{He}$ ions are mentioned: their space resolution is defined as $\delta \mathrm{r}=\mathrm{a} / 10$ ( $\mathrm{a}$ is the minor radius), time resolution is $\delta \tau=100 \mathrm{~ms}$, accuracy between $10-30 \%$. For the neutrons, the space resolution is $\delta \mathrm{r}=\mathrm{a} / 10$, time resolution is $\delta \tau=100 \mathrm{~ms}$, accuracy $10 \%$.

The present paper presents a proposal and motivations to revise the previous measurement requirements for fast particles increasing: i) the spatial resolution ( from a/10 to a/20) corresponding to a scale length close to Larmor radius of alpha particles, and ii) the minimum 
time resolution from $100 \mathrm{~ms}$ to $100 \mu \mathrm{s}-1 \mathrm{~ms}$, this time scale is the saturated non-linerar interaction time of energetic particles with Alfvén modes.

The paper is organized as follows : in Sec 2 the Spatial and temporal scales, and energy spectrum relevant for fast particle detection are presented ; in Sec 3 the requirements for Fast Particle Measurements on ITER and justifications are discussed with the technical characteristics of the candidate systems proposed ; in Sec 4 a summary is presented of the present status and R\&D needed for the diagnostic systems proposed for ITER and concluding remarks are given.

\section{Space, time scales and energy spectrum relevant for fast particle detection.}

Above a critical $\beta_{\text {fast }}$ (beta of fast particles), theoretical analysis predicts that Alfvén cascades can be excited in a reversed shear discharge, giving rise to a spatial redistribution of fast particles over the minor radius in a time scale of $\tau_{\mathrm{F}} \sim 100-300 \tau_{\mathrm{A}} \geq 100 \mu \mathrm{s}\left(\tau_{\mathrm{A}}=\right.$ Alfvén time $=$ $\mathrm{R}_{0} / \mathrm{V}_{\mathrm{A}}, \mathrm{R}_{0}=$ major radius, $\mathrm{V}_{\mathrm{A}}=$ Alfvén velocity). The Alfvén time is calculated using the formula:

$$
\tau_{A}(\mu s)=\frac{0.46 R 0(m) n 20^{0.5} \text { Aeff }}{0.5} ; \quad \quad \text { Aeff }=\frac{n 1 * m 1+n 2 * m 2}{(n 1+n 2) * m H}
$$

Where Aeff is the plasma effective mass referred to the hydrogen $(\mathrm{mH}), \mathrm{n} 20$ is the deuterium density in unities of $10^{20} \mathrm{~m}^{-3}$, R0 the major radius of ITER, B the magnetic field ( Tesla) on axis. For the evaluations of $\tau_{\mathrm{A}}=$ Alfvén time, the following ITER parameters were used: $\mathrm{R} 0=6.2 \mathrm{~m} ; \mathrm{B}=5.2 \mathrm{~T}$; $50 \%-50 \%$ D-T mixture ; $\mathrm{n} 20=0.8$; in the previous formula $\mathrm{m} 1=\mathrm{mD}, \mathrm{m} 2=\mathrm{mT}$, and $\mathrm{n} 1=\mathrm{n} 2, \mathrm{Aeff}=2.5$, and $\tau_{\mathrm{A}}=0.77 \mu \mathrm{s},\left(\mathrm{V}_{\mathrm{A}}=810^{6} \mathrm{~m} / \mathrm{s}\right)$.

To define the measurement requirements for fast particles it is useful to determine the various time scales relevant to ITER: i) time scale related to the saturated non-linear interactions of energetic particles and Alfvén modes [7] $\tau_{\mathrm{F}} \sim 100-300 \tau_{\mathrm{A}}$; ii) slowing down time of the fast ions $\tau_{\mathrm{s}} \sim 1-2 . \mathrm{s}($ see table I) ; iii) confinement time $\tau_{\mathrm{E}} \sim 4 \mathrm{~s}$; iv) resistive relaxation time $\tau_{\mathrm{R}} \sim 200-300 \mathrm{~s}$.

\begin{tabular}{|l|l|l|l|l|l|}
\hline & $\mathrm{m} \alpha / \mathrm{mp}$ & Zeff & $\mathrm{Te}(\mathrm{keV})$ & $\mathrm{ne} / 10^{20}$ & $\tau_{\mathrm{s}}(\mathrm{s})$ \\
\hline alpha & 4 & 1,5 & 30 & 1 & 2,2 \\
\hline $\mathrm{He}^{3}$ & 3 & 1,5 & 30 & 1 & 1,7 \\
\hline $\mathrm{d}$ & 2 & 1,5 & 30 & 1 & 1,1 \\
\hline
\end{tabular}

Table I Slowing down times of fast ions.

The relevant spatial scales in general are: i) Larmor radius $\rho_{\text {fast }} \sim 0.3-7 \mathrm{~cm}$ (see Table IIb); ii) Neoclassical Tearing Mode (NTM) island width $\geq$ ion Larmor radius; iii) Internal Transport Barrier (ITB) width with spatial scale of the order of the pressure gradient; iv) H-mode pedestal width $\sim$ ion Larmor radius; v) turbulence correlation length ion/electron Larmor radius; the scaling of the confinement in $\mathrm{H}$ mode depends upon the Larmor radius. Indeed tomografic reconstruction of $\gamma$-ray spectroscopy on JET , in particular in alpha particle simulation experiments, has demonstrated that the fast particle Larmor radius can be a relevant spatial scale [13]. In general, in absence of instabilities or ITBs, the gradient scale length of the distribution function would be adequate, but in improved confinement scenarios shorter space scales are relevant.

The energy range of fast ion is determined by the critical energy [20] defined as

$$
E^{*}=14.8 T e A_{f}\left(\frac{1}{n e} \Sigma_{j} \frac{n_{j} Z_{j}^{2}}{A_{j}}\right)^{2 / 3}=14.8 T e \frac{A_{f}}{2^{2 / 3}}
$$

where $\mathrm{A}_{\mathrm{f}}$ and $\mathrm{Aj}$ are the atomic masses of fast particle and plasma ions, ne and nj the electron and ion plasma density:the previous formula is valid for a D-T (50\%-50\% composition ). 
The critical energies for fast particles considered for ITER are given in the following table IIa .

\begin{tabular}{|l|r|r|}
\hline & $E^{\star} / T e$ & $A_{f}$ \\
\hline alpha $\left(\mathrm{He}^{4}\right)$ & 37,29 & 4 \\
\hline$T$ & 27,97 & 3 \\
\hline $\mathrm{D}$ & 18,64 & 2 \\
\hline $\mathrm{p}$ & 9,32 & 1 \\
\hline $\mathrm{He}^{3}$ & 27,97 & 3 \\
\hline
\end{tabular}

Table IIa - Critical energies for fast particles

For plasma temperatures $\mathrm{Te}=20 \mathrm{keV}$ ( inductive $\mathrm{Q}=10$, and Weak Reversed Shear scenarios [34]), the range of critical energy for fast particles results in the interval $\mathrm{E}^{*}=180-745 \mathrm{keV}$.

In practice the energy spectrum relevant is $90<\mathrm{E}<1100 \mathrm{keV}$, corresponding to the range $\left[0.5 \mathrm{E}^{*} \min , 1.5 \mathrm{E}^{*} \max \right]$, as it can be seen looking to the fig1, where the slowing-down distribution function $f_{S D}(E) \approx \frac{\left(E / E^{*}\right)^{1 / 2}}{1+\left(E / E^{*}\right)^{3 / 2}}$ is plotted ( see also Table III).

\begin{tabular}{|l|l|l|l|l|l|}
\hline & $\mathrm{mu}=\mathrm{m} / \mathrm{mH}$ & $z$ & $\begin{array}{l}\text { Energy(E) } \\
\text { Temperature(T)(Mev) }\end{array}$ & $\rho(\mathrm{cm})$ & B \\
\hline$\alpha\left(\mathrm{He}^{4}\right)$ & 4 & 2 & $\mathrm{E}=3,50$ & 7,34 & 5,2 \\
\hline$p$ & 1 & 1 & $\mathrm{E}=1,00$ & 1,96 & 5,2 \\
\hline $\mathrm{D}$ & 2 & 1 & $\mathrm{~T}=0,03$ & 0,48 & 5,2 \\
\hline$T$ & 3 & 1 & $\mathrm{~T}=0,03$ & 0,6 & 5,2 \\
\hline $\mathrm{He}^{3}$ & 3 & 1 & $\mathrm{E}=1,00$ & 3,4 & 5,2 \\
\hline
\end{tabular}

Table II Larmor radius of fast ions( Bulk Plasma temperature T=30keV)

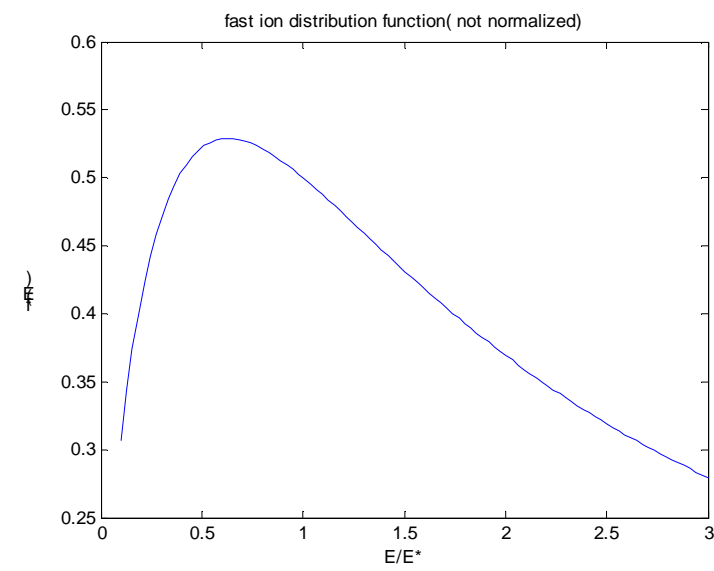

Fig.1 Non-normalized energy distribution function of fast ions( see Table III, and ref 18,20)

In Table III typical parameters of distribution function of fast particles in ITER are listed[18,20]. The nature of the distribution functions(DF) is different for the various fast particles :

i) for alpha particles the DF is an isotropic slowing down

ii) for the other fast ions the DF is anisotropic with the perpendicular velocity higher than the parallel velocity; in both directions ( parallel or hortogonal ) the DF is slowing down. The anisotropy is related to the mechanisms of fast ion tail production by ICRH ( Ion Cyclotron Resonant Heating) . 
The other energy parameter to be taken into account is the resonant energy, i.e. the energy of a particle with velocity equal to the Alfvén velocity (see Table III, column of resonant energy). This parameter is relevant for the wave-particle resonant interaction. For example the Shear Alfven Waves (SAW) are electromagnetic waves satisfying the dispersion relation : $\omega^{2}=k_{p a r}{ }^{2} V_{A}{ }^{2} \cdot\left(\mathrm{k}_{\mathrm{par}}\right.$ is the component of the wavevector along the toroidal magnetic field, $\mathrm{V}_{\mathrm{A}}$ the Alfvén velocity, $\omega / 2 \pi$ the wave frequency). Collective excitations of the SAW spectrum by fast particles are possible since the resonance condition $V_{f}=V_{A}\left(\mathrm{~V}_{\mathrm{f}}\right.$ is the velocity of a fast particle) is likely to be satisfied by the fast ions in ITER plasmas (see Tab III, column of resonant energy). The resonance condition is efficiently maintained, since the SAW group velocity $\vec{V} g(S A W)$ is directed along $\vec{B}$ and has the value $V_{A},\left(\vec{V} g(S A W)= \pm \frac{\vec{B}}{B} V_{A}\right)$ : the fast particles are linked to field lines, moving freely along $\vec{B}$.

Starting from the previous general observations, the new measurement requirements for fast particles could be proposed along the following lines.

i) Spatial resolution. The value of space resolution $\delta r \sim a / 20(10 \mathrm{~cm}$ on ITER) could be taken as reference, because it is close to the alpha particle Larmor radius.

ii) Time resolution. (minimum) $\sim 100 \tau_{\mathrm{A}}(\sim 100 \mu$ s for ITER), which is the time scale related to the saturated non-linear interactions of energetic particles and Alfvén modes[7];

iii) density range of fast ions: the density of minority ions could be $4-10 \%$ of the plasma density

iv) energy spectrum $0.1<\mathrm{E}<3.52 \mathrm{MeV}$,

v) energy resolution of the order of $\geq 10 \%$, if $5-10$ experimental points are measured to trace the slowing-down distribution function( see fig.1).

\begin{tabular}{|c|c|c|c|c|c|}
\hline particles & energy spectrum & $\begin{array}{l}\text { average } \\
\text { density }\left(10^{20} \mathrm{~m}^{-3}\right)\end{array}$ & $\beta$ fast $/ \beta$ total $(\%)$ & \begin{tabular}{|l} 
Resonant \\
Energy \\
$(\mathrm{MeV})$ \\
\end{tabular} & source \\
\hline alpha & $\begin{array}{l}\mathrm{f}(\mathrm{E}) \mathrm{SD} \sim \mathrm{E}^{1 / 2} /\left(\mathrm{E}^{3 / 2}+\mathrm{E}^{\star 3 / 2}\right) \\
\mathrm{E}^{*}=20 \mathrm{Te} ; 0.1<\mathrm{E} \leq 3.52 \mathrm{MeV} \\
\text { isotropic }\end{array}$ & $0.7510^{-3}-2.510^{-2}$ & 10 to 20 & 1.35 & D-T fusion reactions \\
\hline $\mathrm{D}$ or $\mathrm{H}$ & $\begin{array}{l}\text { Anisotropic;Vperp>Vpar; } \\
E<1 \mathrm{MeV} ; f(E)=f(E) S D\end{array}$ & 2-4 $10^{-3}$. & 3 to 7 & $\begin{array}{l}E D=0.67 \\
E H=0.33\end{array}$ & NB heating \\
\hline $\mathrm{T}$ & $\begin{array}{l}\text { Anisotropic;Vperp }>\text { Vpar; } \\
E<0.2-0.3 \mathrm{MeV} ; \\
f(E)=f(E) S D\end{array}$ & & & 1 & $\begin{array}{l}2 \omega C T \text { ICRF heating in D- } \\
\text { T plasma }\end{array}$ \\
\hline $\mathrm{He} 3$ & $\begin{array}{l}\text { Anisotropic; } \\
\text { Vperp>>Vpar;E 0.1-1MeV }\end{array}$ & $4-1010^{-2}$ & $<10$ & 1 & ICRF minority heating \\
\hline
\end{tabular}

Table III Typical parameters of fast ions in ITER(see also ref 18).

\section{Requirements for Fast Particle Measurements on ITER and justifications}

The previous analysis leads to the measurement requirements of fast particles that are listed in Table IV, where a number of potential electron ( see sec 3.5) and wave diagnostics are included. 


\begin{tabular}{|c|c|c|c|c|c|c|c|c|}
\hline particles & $\begin{array}{l}\text { requested } \\
\text { measurement }\end{array}$ & group & techniques & $\begin{array}{l}\text { spatial } \\
\text { resolution }\end{array}$ & \begin{tabular}{|l} 
time \\
resolution
\end{tabular} & accuracy & $\begin{array}{l}\text { energy } \\
\text { spectrum(MeV) }\end{array}$ & $\begin{array}{l}\text { density } \\
\text { interval } \\
\left(10^{18} \mathrm{~m}^{-3}\right) \\
\end{array}$ \\
\hline $\begin{array}{l}\alpha, p, D, T, \\
\mathrm{He}^{3} .\end{array}$ & $\begin{array}{l}\text { spatial and } \\
\text { energy } \\
\text { distribution }\end{array}$ & $\begin{array}{l}\text { advanced } \\
\text { control }\end{array}$ & $\begin{array}{l}\gamma \text {-ray } \\
\text { spectr.;CTS;CXRS; } \\
\text { NPA(*); passive } \\
\text { spectroscopy(Line } \\
\text { ratio) }\end{array}$ & $a / 20$ & $0.1-0.2 \mathrm{~s}$ & $10 \%$ & $\begin{array}{l}E_{\alpha}=0.1-3.5 \\
E H e^{3}=0.1-1\end{array}$ & $\begin{array}{l}\mathrm{n}_{\alpha}=0.075 \\
2.5 \\
\mathrm{nHe}^{3}=4- \\
10\end{array}$ \\
\hline & $\begin{array}{l}\text { fast particle } \\
\text { losses }\end{array}$ & $\begin{array}{l}\text { advanced } \\
\text { control }\end{array}$ & $\begin{array}{l}\text { Faraday cups, } \\
\text { scintillator probes; } \\
\text { ceramic } \\
\text { scintillators; } \\
\text { IRMFTD( }\left(^{\star}\right) ; \\
\text { activation } \\
\text { foils;IRV }\left(^{\star}\right) \\
\end{array}$ & & $0.1-0.5 \mathrm{~ms}$ & $15 \%$ & & \\
\hline neutrons & $\begin{array}{l}\text { Energy } \\
\text { spectrum at } \\
2.5 \text { and } \\
14 \mathrm{MeV}\end{array}$ & $\begin{array}{l}\text { advanced } \\
\text { control }\end{array}$ & $\begin{array}{l}\text { NE213 scintillator, } \\
\text { CVD and NDD } \\
\text { compact } \\
\text { spectrometers, } \\
\text { fission chambers }\end{array}$ & $a / 20$ & $0.1-0.5 \mathrm{~ms}$ & $10-15 \%$ & & \\
\hline electrons & $\begin{array}{l}\text { spectrum of } \\
\text { ECE }\end{array}$ & $\begin{array}{l}\text { physics } \\
\text { evaluation }\end{array}$ & Michelson, Thomson & a/20 & $1 \mathrm{~ms}$ & $10 \%$ & & \\
\hline $\begin{array}{l}\text { FW,IBW } \\
\text { and AE }\end{array}$ & $\begin{array}{l}\text { Phase } \\
\text { contrast } \\
\text { imaging }\end{array}$ & $\begin{array}{l}\text { physics } \\
\text { evaluation }\end{array}$ & $\begin{array}{l}\text { Phase contrast } \\
\text { imaging using } \mathrm{CO} 2\end{array}$ & $a / 40$ & $0.1 \mathrm{~ms}$ & & & \\
\hline
\end{tabular}

Table IV - Requirements for measurements on fast particles and candidate techniques on ITER.

(*) CTS=Collective Thomson Scattering;CXRS=Charge exchange recombination spectroscopy;NPA=Neutral particle analyzers; IRMFTD $=I R$ multifoil thermal detectors; $I R V=I R$ videocam

\subsection{Fast particle measurement requirements and justifications}

The measurement requirement on the energy spectum of confined alphas is $0.1<\mathrm{E} \alpha<3.5 \mathrm{MeV}$. For the other fast ions it is $0.1<\mathrm{E}<1 \mathrm{MeV}$. The spatial resolution should be $10 \mathrm{~cm}$, and temporal resolution $100 \mathrm{~ms}$, for all fast ions present in the plasma.

The space resolution corresponds to a spatial scale of the Larmor radius because the spatial dynamics of distribution functions related with the interaction of fast particles with Alfvén waves is the Larmor radius .The necessity of having a spatial resolution of $a / 20$ is related to the introduction of measurements on all the fast ions. The Larmor radius of fast ions is in the range of $0.5-7 \mathrm{~cm}$, and the proposed space resolution $(\mathrm{a} / 20=10 \mathrm{~cm}$ for ITER) is still an upper limit for most of fast ions.

The proposed Time resolution is related to a fraction $(=1 / 10)$ of the minimum slowing down time of fast particles ( see Table I).

Ideally the shortest time resolution related to $\tau_{\mathrm{F}} \sim 100-300 \tau_{\mathrm{A}} \sim 0.1 \mathrm{~ms}$ (the time scale of saturated nonlinear interactions of fast particles and Alfvén modes) should be included in the measurement of the energy distribution function of fast particles, if the fast spatial redistribution due to Alfvén modes is needed to be detected. At moment this time resolution seems an hard limit for the most important techniques ( $\gamma$-ray spectroscopy, Collective Thomson Scattering, Charge Exchange Recombination Spectroscopy) useful to measure the distribution function of confined fast particles.

\subsection{Fast particle Diagnostic systems}

\subsection{1. $\gamma$-ray spectroscopy}

This technique allows the detection of ions in the energy range $1<\mathrm{E}<5 \mathrm{MeV}$ [13]. Alphas are detected using $\mathrm{Be}$ as impurity: ${ }^{9} \mathrm{Be}(\alpha, \mathrm{n} \gamma){ }^{12} \mathrm{C}$ and $\gamma$-rays with energy $\mathrm{E} \gamma=4.44 \mathrm{MeV}$.Deuterons are 
detected using ${ }^{12} \mathrm{C}(\mathrm{d}, \mathrm{n} \gamma){ }^{13} \mathrm{C}$, $\gamma$-rays with energy $\mathrm{E} \gamma=3.1 \mathrm{MeV} . \mathrm{He}^{3}$ is detected using ${ }^{12} \mathrm{C}\left(\mathrm{He}^{3}, \mathrm{n} \gamma\right){ }^{14} \mathrm{~N}$ $\gamma$-rays with energy $\mathrm{E} \gamma=2.31$ and 5.1MeV.R\&D is needed to demonstrate whether the neutron background can be rejected by $\mathrm{LiH}$ absorbers. In practice ( looking to JET experience ) the required accuracy is difficult to achieve. The integration time needed is in the range of $100 \mathrm{~ms}$. So the gamma ray spectroscopy could see the slowing down of fast ions, but not the fast spatial redistribution due to the interaction with Alfvén waves.

\subsubsection{Collective Thomson Scattering(CTS)}

The CTS proposed [19] uses a gyrotron at $60 \mathrm{GHz}$, applying the principle [21] that using a source at a frequency below the first harmonic of ECE is beneficial to lower the plasma background temperature, definitely increasing the signal to noise ratio. The system proposed has the capability of measuring ions with velocity parallel and perpendicular to the magnetic field.The spatial resolution is $20 \mathrm{~cm}$ (i.e. a/10) and the time resolution is $100 \mathrm{~ms}$, with an accuracy of $20 \%$. The main difficulty of CTS is that in principle it cannot distinguish between ions with the same Z/M. The method foreseen for ITER is focused on detecting alpha particles, but has only limited capability to diagnose other fast particles. CTS nominally meets requirements, but the spatial resolution cannot be met within the requirement of $100 \mathrm{~ms}$. $R \& D$ is needed to test the ITER concept in a pilot experiment. CTS Experiment on FTU [21] includes the basic ITER concept ( working below the first ECE harmonic) and it is aimed at the measurement of ion temperature.

\subsubsection{CXRS}

Various authors[22,23] have given estimates for the possibility of measuring the alpha particles , and fast ions using CX recombination lines. Analysis reported in ref [22] leads to a signal to noise ration $\mathrm{SNR}=7$ at $\mathrm{r} / \mathrm{a}=0.3$ for $100 \mathrm{~ms}$ integration time and $\mathrm{ne}=110^{20} \mathrm{~m}^{-3}$ for alpha particles . The evaluation for fast deuterons leads to a $\mathrm{SNR}=60$ at $\mathrm{r} / \mathrm{a}=0.3$ in the same conditions, using the heating beam(HB), HB4 and HB5. The lines used in the evaluations are at $\lambda=468.6 \mathrm{~nm}$ for alphas , and $\lambda=656.1 \mathrm{~nm}$ for deuterons.Similar evaluations were presented in ref [23] : the diagnostic neutral beam (DNB) could be used for the measurements of low energy alpha particles $100<\mathrm{E}_{\alpha}<600 \mathrm{keV}$, achieving a SNR=5-10 at $\rho=0.3$; while the heating neutral beam HNB5 could be used for the measurement of alphas $1600<\mathrm{E}_{\alpha}<2400 \mathrm{keV}$, achieving a $\mathrm{SNR}=3-5$ at $\rho=0.3$. In principle it would be possible to measure the spectrum of fast ions:i) the integration time will be $100 \mathrm{~ms}$; ii) the spatial resolution will be $>\mathrm{a} / 20$. The capability of measuring the fast ions by CXRS close to the plasma centre must be still demonstrated using the DNB and/or the HB. Recently the concept [24] of using $\mathrm{a}^{3} \mathrm{He}^{0}$ diagnostic beam ( Energy $1.7 \mathrm{MeV}$ ), has been proposed based on a double charge exchange to detect the fast $\mathrm{He}$ in the range $1<\mathrm{E}<3 \mathrm{MeV}: \mathrm{He}^{++}$(plasma) $+\mathrm{He}^{0}($ beam $) \Rightarrow \mathrm{He}^{0}$ (plasma $)+$ $\mathrm{He}^{++}$. The calculated signal to noise ratio is encouraging $(\mathrm{SNR}=10)$ at plasma centre. $\mathrm{R} \& \mathrm{D}$ is needed to demonstrate the feasibility of the $\mathrm{He}^{0}$ beam and the SNR calculated for the detection of fast ions, in a dedicated pilot experiment. It is not clear whether such a system could be implemented on ITER.

\subsubsection{Passive spectroscopy}

As it was mentioned above, the energy range $200<\mathrm{E}<800 \mathrm{keV}$ is relevant for the study of the interaction between the fast particles and Alfvén waves ( see Table III, and fig.1). The diagnostic capability of fast ions in this energy range is weak because the only proven technique to measure the fast particles, i.e. $\gamma$-ray spectroscopy, is not useful in this interval. A new technique is now under test on JET [25], using the sensitivity to fast particles of fluorine like configurations of extrinsic impurities like Krypton. It is found that the transition $n=2$ to $n=1$ of KryptonXXVIII ( which is obtained starting from $n=1$, via the transition to the $n=3$ level, and subsequent decay to the $n=2$ ) depends on the fast ion population. Preliminary results of experiments on JET, in a power scan using the $80 \mathrm{keV}$, Deuterium Neutral Beam of max power of $8 \mathrm{MW}$, it was found that the 
ration between the line at $\lambda=22.4 \mathrm{~nm}$ and that at $\lambda=5.259 \mathrm{~nm}$ of $\mathrm{KrXXVIII}$ was dependent upon the NBI power, which is proportional to the fast particles injected in the plasma.

\subsubsection{Neutral Particle Analyzer(NPA).}

A prototype compact tandem system including high energy ( $\mathrm{E}=0.1-4 \mathrm{MeV}$ ) as well as low energy(10-200keV) NPA was developed for ITER [26]. Studies of electromagnetic and neutron shielding are in progress.

\subsubsection{Lost fast particle diagnostics.}

Faraday Cups (FC) and Scintillator probes(SP) were already tested on JET [27] and TFTR[28]. Presently a new system of FC and SP is under commissioning at JET, and it could be proposed for ITER. The JET FC system measures the poloidal distribution of lost fast ions with a course energy resolution. Alpha particles in the range of 1-3 MeV can be detected with an energy resolution of the order of $10-15 \%$. The SP measures the gyroradius and pitch angle of a fast particle, the accuracy of measurement of gyroradius is $15 \%$, while that of the pitch angle is $5 \%$, the time resolution is $0.1 \mathrm{~ms}$. In practice a JET-like SP system could meet the requirement on the measurement, if a way to integrate such a system on ITER is found: systems as scintillators and Faraday-cups are subject to failure in the high radiation fields at ITER. Preliminary analysis lead to consider other systems like ceramic scintillators and infrared multifoil thermal detectors( see ref.33)

\subsection{Neutron measurement requirements.}

The measurement of the spatial profile of neutron emission with a spatial resolution of $10 \mathrm{~cm}$ is required. The time resolution required is $0.1-0.5 \mathrm{~ms}$. Vertical and radial neutron cameras are required, because the radial movement of fast ion interacting with Alfven waves could be detected only having vertical as well as horizontal cameras. The spatial resolution is set to alpha particle Larmor radius, and the time resolution is related with the saturated non-linear time of interaction between fast ions and Alfven eigenmodes in shear reversed scenario. An accuracy of $15 \%$ is set for these measurements. The neutron camera is supposed to be equipped with compact neutron spectrometers for $2.5 \mathrm{MeV}$ and $14 \mathrm{MeV}$, tested during the Trace Tritium Experiment on JET and based on NE213 scintillators [29]. A fast electronics is needed for the neutron camera to discriminate the neutrons from gammas, and to achieve the required time resolution: this electronics is under test at JET, and preliminary results are encouraging.

\subsection{Neutron diagnostic systems.}

\subsubsection{Neutron Camera.}

A study [30] has been done about the possibility for the ITER neutron camera to meet the requirements. Parameters assumed in the study: DT full power (scenario 2), diameter of collimators $2 \mathrm{~cm}$ in-vessel and $1 \mathrm{~cm}$ ex-vessel (corresponding to 1-2 $\mathrm{MHz} \max$, count rate sustainable by the detectors), $0.1 \mathrm{~ms}$ time resolution, detector efficiency $1 \%$.The code used for the design of the ITER RNC has produced the Abel inverted neutron emissivity, assuming the equilibrium of ITER, the emissivity to be constant on the magnetic surfaces and the actual configuration of the line of sights of the ITER RNC. For the case of $0.1 \mathrm{~ms}$ time resolution, the result is that the accuracy on the emissivity is inside $20 \%$. In practice it is reasonable to assume that the ITER neutron camera could be close to the technical specifications. In this context is worth mentioning that strong asymmetries were found in the neutron emission in JET TTE [see ref 1, fig14], in particular in strong shear reversed discharges ( so called current-hole) configurations. These results lead to the need for ITER of a vertical neutron camera(VNC). The measurement requirements reported in Table IV could be obtained only with the use of a VNC.

\subsubsection{Neutron compact spectrometers.}


Compact Spectrometers(CS) based on NE213 scintillator [30] were tested in JET TTE and a reasonable energy resolution was obtained of $\Delta \mathrm{E} / \mathrm{E} \sim 2 \%$ for neutrons of $14 \mathrm{MeV}$ and $\Delta \mathrm{E} / \mathrm{E} \leq 4 \%$ for 2.5MeV neutrons. CS based on CVD ( Carbon Deposited Diamond) are under development [31] an energy resolution close to $1 \%$ for $14 \mathrm{MeV}$ neutrons seems feasible for these systems.

\subsection{Measurements of the electron distribution function(EDF).}

The EDF measurements are linked with the effects of the heating ( including alpha particles produced by the fusion reactions ) and current drive systems on electrons. The fast electron bremmsstrahlung was used to monitor the high energy tail of fast electrons produced by the Lower hybrid heating[35] through the hard X spectra. Thomson scattering is the technique which measures directly the EDF. The measurement of the ECE is proposed at various angles with respect to the magnetic field to measure the possible deviations of the electron distribution function from a maxwellian. The motivation is related with the consequences of this deviation on the measurements of plasma temperature using ECE only. This topic has been addressed in particular in coincidence with high temperature $\mathrm{Te}>7 \mathrm{keV}$ [32] where there is a difference in the measurements of Te. The Michelson interferometer is a system which is suitable for meeting the requirement in table IV. The accuracy ( statistical + systematic errors) on the measurements of electron temperature needed for detecting effects of deviations from maxwellian must be very high $(<7 \%)$ for both ECE and TS measurements, and this is an objective quite demanding to achieve.

\section{Summary of present status of diagnostic systems proposed for ITER, R\&D needed and conclusive remarks.}

The candidate diagnostic techniques considered for ITER are: i) $\gamma$-ray spectroscopy; ii) Collective Thomson Scattering (CTS); iii) Charge Exchange Recombination Spectroscopy (CXRS). $\gamma$-ray spectroscopy detects ions in the energy range $1<\mathrm{E}<5 \mathrm{MeV}$, and $\mathrm{R} \& \mathrm{D}$ is needed to demonstrate the feasibility of these measurements( gamma ray) in the presence of a neutron background. Techniques using ultraviolet spectroscopy of ions like Krypton are being tested for the low energy part of spectrum of fast particles. A design study of CTS shows that the requirements are close to be met, the system includes a high power gyrotron operating at a frequency below the first ECE harmonic. A system exploring this configuration is mounted on FTU. For CXRS the spatial resolution and accuracy are not achievable for $r / a<0.3$, and $R \& D$ is needed to assess the minimum figures in terms of accuracy possible for measurements close to the centre. The diagnostics for measuring fast particle losses (i.e. faraday cups and scintillator probes) are difficult to implement on ITER.In practice the minimum time resolution of $100 \mu \mathrm{s}$ is difficult to achieve, in this context, also using IR multifoil thermal detectors with alpha absorbers, and activation foil techniques. The measurement of the neutron and alpha source profile measurements is done by two neutron cameras: a horizontal and vertical camera. In the neutron camera compact spectrometers as well as $\gamma$-ray spectrometers could be inserted. The vertical camera is important for detecting fast movements of the energetic ions.

The diagnostics of the electron distribution function could be achieved inside the required technical specifications by the present technology. In particular a Michelson interferometer with oblique views could be used to measure the behaviour of the electron distribution function.

Measurements of plasma waves by phase contrast imaging using $\mathrm{CO}_{2}$ laser could give some important information on Alfvén cascades. The existing projects of reflectometers on ITER could include the capability of measuring waves and MHD activity related with fast ions.

The main message of this paper is that the development of fast particle diagnostics is a real challenge: the measurement requirements deduced from the need of detecting the effects of interaction of fast particles with MHD instabilities put important new objectives to the candidate diagnostic systems. In particular diagnostics for fast particles in the low energy region 
$100<E<1000 \mathrm{keV}$ meeting the requirements of table $\mathrm{IV}$, and the diagnostics for escaping fast particles need a urgent development.

\section{Acknowledgements}

One of the Authors(FO) would like to acknowledge the support of EFDA. F Zonca is acknowledged for discussions on theory of fast particle dynamics and the requirements on the measurements. D Borba, W Heidbrink, A Fasoli are gratefully acknowledged for very useful comments.

\section{References}

1. D Stork et al - Nucl Fus 45(2005)S181.

2. S Pinches et al PPCF 46(2004)B187

3. S Sharapov et al Proc $31^{\text {st }}$ EPS Conf. on Plasma Physics(London 2004) P5-166

4. K Shinohara et al. PPCF 46(2004)S31-S45

5. M Ishikawa et al Nucl Fus 46(2006)S898-S903

6. L Chen Phys Plasmas 1(1994)1519

7. G Vlad et al in Fusion Energy 2006 ( Proc $21^{\text {st }}$ Int Conf Chengdu, 2006) ( Vienna:IAEA) CD-ROM file TH/P6-4 and http://wwwnaweb.iaea.org/napc/physics/FEC/FEC2006/html/index.htm

8. R Nazikian et al PRL 78(1997)2976

9. J Jacquinot et al Nucl Fus 38(1998)1263

10. J A Snipes et al Phys Plasmas 12(2005)056102

11. M Porkolab et al - IEEE Trans Plasma Science 34(2006) 229

12. A J H Donne et al in Fusion Energy 2000(Proc. 18th Int. Conf. Sorrento,2000)(Vienna:IAEA) CD-ROM file[CN-77/ITERP/09] and http://www.iaea.org/programmes/ripc/physics/fec2000/html/node1.htm

13. V G Kiptily et al Nucl Fus 45(2005) L21-L25

14. S Hacquin et al Nucl Fus 46(2006) S714

15. W.Heidbrink et al - PPCF 46(2004)1855

16. M Sasao et al PPCF 46(2004)S107-S118

17. JET Team , PPCF 37(1995)A3(fig.15).

18. S V Putvinski Nucl Fus 38(1998)1275

19. H Bindslev et al - Phys Rev Lett 97(2006) 205005

20. J Wesson - Tokamaks , $2^{\text {nd }}$ Edition, Oxford Science Publications 1997

21. F Orsitto and G Giruzzi Nucl Fus 37(1997)915;

22. S Tugarinov et al - Rev Sci Instr 74(2003) 2075

23. M von Hellerman et al. - in IAEA FEC 2006 Chegdu - paper IT/P1-26.

24. M Sasao et al. - Rev Sci Instr 77(2006)10F130

25. A Murari et al.- Rev Sci Instr 77(2006) 10F529

26. B Lyublin $-8^{\text {th }}$ ITPA TG Dia Meeting Culham(UK) march 2005

27. G J Sadler et al Nucl Fus 35(1995)1609

28. S J Zweben et al Nucl Fus 40(2000) 91

29. L Bertalot et al Report EFDA-JET CP(05)-02-17

30. L Petrizzi et al. Soft $2006-24^{\text {th }}$ Symposium on Fusion Technology- Poster P1-D-455.

31. M Marinelli et al App Phys Lett 89(2006)1

32. E De la Luna et al . EFDA-JET Report CP(02)03/10 (HTPD Madison 2002).

33. T Donné et al - in Fusion Energy 2006 ( Proc $21^{\text {st }}$ Int Conf Chengdu, 2006) ( Vienna:IAEA) CD-ROM file IT/P1-24 and http://wwwnaweb.iaea.org/napc/physics/FEC/FEC2006/html/index.htm

34. B J Green et al. PPCF 45(2003)687

35. Y Peysson and F Imbeaux RSI 70(1999)3987 